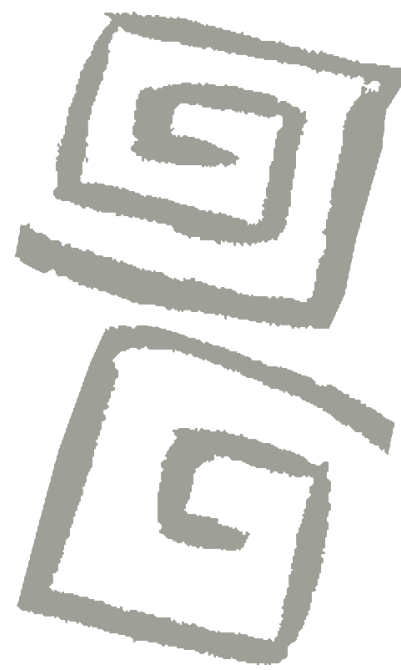

\title{
Condiciones de trabajo y salud en docentes universitarios y de enseñanza media de Mendoza, Argentina: entre el compromiso y el desgaste emocional
}

\author{
Health and working conditions of high school and \\ university teachers in Mendoza: between commitment \\ and emotional distress
}

\author{
Patricia Alejandra Collado ${ }^{1}$, Cecilia Beatriz Soria², Eliana Canafoglia ${ }^{3}$, Sandra \\ Alicia Collado ${ }^{4}$
}

'Licenciada en Sociología, Doctora en Ciencias Políticas y Sociales. Investigadora Adjunta Consejo Nacional de Investigaciones Científicas y Técnicas (CONICET), Profesora e investigadora, Facultad de Ciencias Políticas y Sociales, Universidad Nacional de Cuyo, Mendoza, Argentina. $\square$ (iD)

¿2icenciada en Sociología, Doctora en Ciencias Sociales. Facultad de Ciencias Políticas y Sociales, Universidad Nacional de Cuyo, Mendoza, Argentina. $\square$ (iD

${ }^{3}$ Licenciada en Sociología Doctora en Ciencias Sociales, Becaria Posdoctoral, Consejo Nacional de Investigaciones Científicas y Técnicas (CONICET). Profesora e investigadora, Facultad de Ciencias Políticas y Sociales, Universidad Nacional de Cuyo, Mendoza, Argentina. $\square$ (iD)

${ }^{4}$ Licenciada en Calidad, Medio Ambiente e Higiene y Seguridad en el Trabajo, Doctoranda en Ciencias Políticas y Sociales, Facultad de Ciencias Políticas y Sociales, Universidad Nacional de Cuyo, Mendoza, Argentina. $\square$ (iD
RESUMEN Con el objetivo de analizar los aspectos vinculados con la percepción sobre las condiciones de trabajo y su impacto en la salud de los docentes que pertenecen a la Universidad Nacional de Cuyo (UNCuyo), Mendoza, Argentina, este artículo analiza los resultados del Primer Censo de Condiciones y Salud Laboral, realizado a fines del año 2013, en dos unidades académicas (una de enseñanza media y otra de nivel universitario) que abarcó a 193 docentes. El sondeo se dedicó a caracterizar el plantel de trabajadores/as y las condiciones que afectan su salud, principalmente en sus aspectos psicosociales, para lo cual se aplicó un cuestionario autoadministrado, cuyas dimensiones fueron debatidas en talleres de sensibilización con docentes que colaboraron en la preparación del instrumento de recolección. Entre sus principales resultados emerge el desgaste físico y emocional de trabajadores altamente calificados, por el efecto combinado de la respuesta comprometida a las exigencias de su labor con la degradación (material y simbólica) de las condiciones en que la desempeña.

PALABRAS CLAVES Salud Laboral; Condiciones de Trabajo; Docentes; Instituciones Académicas; Argentina.

\begin{abstract}
With the objective of analyzing aspects related to the perception of working conditions and their impact on health in the teachers and professors who work for the Universidad Nacional de Cuyo (UNCuyo) in Mendoza, Argentina, this work analyzes the results of the Primer Censo de Condiciones y Salud Laboral [First Census on Health and Working Conditions]. The census was conducted in late 2013 in two academic units (one at the high school level and the other at the university level), including 193 educators. The exploration set out to characterize the teaching staff and the conditions affecting their health, primarily with respect to psycho-social health. In order to do, so a self-administered questionnaire was applied, the dimensions of which were discussed in sensitivity workshops with educators who helped to formulate the data collection instrument. Among the primary results emerge the physical and emotional burnout of these highly skilled workers, owing to the combined effect of their committed response to the demands of their work and the deterioration (both material and symbolic) of the conditions in which they carry out that work.
\end{abstract}

KEY WORDS Occupational Health; Working Conditions; Faculty; Schools; Argentina. 


\section{INTRODUCCIÓN}

El ámbito laboral educativo está atravesado por fuertes transformaciones que trascienden y abarcan al conjunto del mundo del trabajo, particularmente, a todas las actividades que podemos nominar como asalariadas de servicios.

Estas mutaciones inciden profundamente en las condiciones, situación y salud de los trabajadores, las cuales se relacionan con múltiples factores, entre ellos: los avances en el campo tecnológico y su asociación con el trabajo humano ${ }^{(1)}$, las transformaciones en el proceso y los contenidos del trabajo a escala global $^{(2)}$ y los cambios en las relaciones laborales, en cuyo establecimiento predomina la precariedad e inestabilidad ${ }^{(3)}$.

En este marco, la labor docente destaca por sus especificidades. Sus características se relacionan tanto con la degradación de la cuestión social en general ${ }^{(4)}$ como con las mutaciones del trabajo ya mencionadas, potenciando el desgaste de la fuerza laboral docente.

El trabajo educativo, como productor de un bien inmaterial que es "incorporado" a la subjetividad de su destinatario ${ }^{(5)}$, padece fuertemente el deterioro social debido, en gran medida, a la pauperización y vulnerabilidad de la condición socioeconómica de los educandos y educadores, y a sus consecuencias: el menoscabo de las relaciones sociales y la fragmentación vincular. A la cuestión social, se suma la crisis y el deterioro de los bienes públicos que se evidencia en el desgaste de la infraestructura y los medios de trabajo en general ${ }^{(6)}$. En este escenario, y como correlato de la intangibilidad de su obrar, la forma de asegurar la productividad de los docentes, los contenidos y la orientación de su trabajo se encamina a exacerbar los controles sobre sus tareas, a desarrollar múltiples y superpuestos mecanismos de evaluación de desempeño y a multiplicar las exigencias. Todas estas cuestiones adoptan un cariz singular en cada formación social ${ }^{(7,8,9,10)}$.

En Argentina, la gran transformación de las condiciones de trabajo se legitimó a partir de los años noventa con la reforma del empleo, a través de un cúmulo de normativas que desregularon el mercado y flexibilizaron la fuerza laboral ${ }^{(11)}$, cambiaron estructuralmente el derecho protectorio del trabajo ${ }^{(12)}$, promovieron la inserción laboral precaria ${ }^{(13)}$ e intensificaron el trabajo ${ }^{(14)}$.

La situación específica de los docentes universitarios puede comprenderse a partir del nuevo modelo educativo que propició la Ley Federal de Educación de 1993, con implicancias profundas para la labor docente que persisten aun luego de su derogación ${ }^{(15,16)}$. La descentralización salarial, el Programa de Incentivos a Docentes-Investigadores vinculado a la productividad académica y las nuevas formas de evaluación y control de la información sobre el plantel de trabajadores son algunas de sus consecuencias más notorias. Esta normativa permitió, centralmente, flexibilizar el vínculo laboral al posibilitar la implementación de diversas modalidades contractuales: contratos a término, facturación como cuenta propia o por servicios, pasantías, entre otras. De este modo, se extendió la inestabilidad del plantel docente bajo la forma masificada de los interinatos, como un indicador fehaciente de la precariedad de los universitarios ${ }^{(17)}$.

En cuanto a los cambios en los contenidos del trabajo docente, estos se expresan en la consecución de perfiles ensamblados y polifacéticos que impusieron la polifuncionalidad, bajo la figura del docente-investigador ${ }^{(10)}$; la sobreocupación por acumulación de cargos, puestos laborales, tareas que se acopian por desmejora de los niveles salariales ${ }^{(18,19)}$ y las evaluaciones de desempeño que favorecen la meritocracia y exacerban la competitividad al interior del colectivo laboral ${ }^{(20)}$. Finalmente, el impacto tecnológico con relación a la labor docente se manifiesta en las tareas de gestión y administración del plantel de trabajadores y la evaluación de su productividad en términos de desempeño, adoptando un cariz de dispositivo de control ${ }^{(21,22)}$. Es decir, dicho impacto no se vincula de manera directa con los contenidos de la labor educativa o pedagógica ${ }^{(23)}$.

De lo anteriormente dicho surge la necesidad de complejizar las investigaciones 
sobre condiciones de trabajo, sin restringirlas a las condiciones materiales en las cuales los trabajadores realizan sus labores o -en una versión ampliada- a evaluar las cargas globales del trabajo ${ }^{(24)}$. Sin desmerecer estos aspectos, tal como vimos, cada sociedad, momento histórico y ámbito laboral merecen ser apreciados en su singularidad para evaluar los desgastes y usos de la fuerza laboral. Así acontece con los docentes universitarios.

Este escrito es producto del proceso de investigación participativa que realizamos con los docentes de la Universidad Nacional de Cuyo, en dos establecimientos educativos para caracterizar uno de los aspectos que impacta en su condición laboral: la salud psicosocial.

Los factores psicosociales son aquellas características de las condiciones de trabajo y, sobre todo, de su organización que afectan a la salud de las personas a través de mecanismos psicológicos y fisiológicos a los que también Ilamamos estrés. En términos de prevención de riesgos laborales, los factores psicosociales representan la exposición (o los factores de riesgo), la organización del trabajo, el origen de ésta, y el estrés el precursor del efecto (enfermedad o trastorno de salud que pueda producirse $y$ que debemos prevenir) ${ }^{(25}$ p.14)

Para alcanzar tal fin, partimos de una concepción sobre la persona como entramado biográfico de múltiples relaciones y comprendemos la salud-coincidiendo en parte con la Organización Internacional del Trabajo (OIT) - como bienestar físico, mental y social ${ }^{(26)}$. Debido a esta posición teórica, la salud laboral asume un fuerte componente social, ya que el bienestar de cada trabajador está atravesado por las formas en que efectivamente realiza el trabajo y los modos diversos en que la actividad los afecta. Es decir, la salud puede verse desmejorada, atenuada o impactada por factores que se asocian a las formas de hacer, ser y estar en el trabajo.

En esta perspectiva, los factores de riesgo psicosocial poseen dos aristas. Por un lado, se encuentran aquellos relacionados con la organización y los contenidos del trabajo que componen el aspecto "social" del riesgo $y$, por el otro, los relativos a efectos sobre la salud. En esta línea de investigación existen innumerables antecedentes, entre los más difundidos se encuentran los que vinculan estrés laboral y burnout ${ }^{(27,28,29,30,31)}$. Para dar cuenta de los aspectos psicodinámicos del trabajo, nos apoyamos en Christophe Dejours $^{(32,33)}$, a fin de considerar la relación entre condiciones de trabajo y la generación de sufrimiento mental. También nos respaldamos en la obra de Christian Baudelot y Michel Gollat ${ }^{(34)}$ para comprender la satisfacción laboral, la felicidad e infelicidad en el trabajo e incluimos aportes de Marie France Hirigoyen $^{(35)}$ e Iñaqui Piñuel(36) y de autores argentinos compilados en la obra de Viviana Vega ${ }^{(37)}$, sobre todo en lo referido al acoso laboral y tramas vinculares. Es importante destacar que nuestra perspectiva de abordaje se sostiene en la sociología crítica del trabajo como mirada disciplinar central. En este sentido, el enfoque privilegia lo social, sin desmerecer el reconocimiento de la subjetividad como productora y producida por múltiples tramas de relaciones vinculares.

\section{CONSIDERACIONES METODOLÓGICAS}

Con el objetivo de analizar los aspectos vinculados con la percepción sobre las condiciones de trabajo y su impacto en la salud de los docentes que pertenecen a la Universidad Nacional de Cuyo realizamos el Primer Censo de Condiciones y Salud Laboral Docente.

Dicha universidad es la principal institución pública de educación superior de la región centro-oeste de Argentina. Se fundó en el año 1939 y actualmente cuenta con 15 facultades e institutos, 111 carreras, seis colegios preuniversitarios y un colegio primario. En 2013, contaba con 32.425 estudiantes mientras que el plantel docente se distribuía en 3.865 en el nivel universitario y 1.230 en el nivel secundario(38,39). 
Por tanto, la primera fase del mencionado censo, realizado en el año 2013, se relevaron dos unidades académicas. Una de ellas corresponde al nivel universitario (cuyas áreas de formación son la industria química, de alimentos y vitivinícola), en tanto la segunda pertenece al nivel de enseñanza media (orientada a la formación docente), ambas dependientes de dicha casa de estudios.

Se trata de un estudio descriptivo sobre un total de 193 docentes, de los cuales 78 eran profesores universitarios y 115 de educación media, que representaron el $74 \%$ y $77 \%$ del plantel docente activo durante el relevamiento en las unidades académicas seleccionadas. La selección de estas unidades académicas para esta primera fase respondió a requerimientos realizados por la organización gremial.

El diseño del instrumento se realizó mediante revisión y evaluación de estudios y fuentes que han abordado las condiciones de trabajo y la promoción de la salud de los trabajadores. El análisis de estas investigaciones y de los cuestionarios aplicados nos posibilitó determinar dimensiones, que fueron resignificadas a la luz de nuestra perspectiva teórica y las especificidades de la población bajo estudio. Las principales fuentes consultadas fueron:

- ISTAS21 $1^{(25)}$, cuestionario adaptado por el Instituto Sindical de Trabajo, Ambiente y Salud (ISTAS) de España, del Copenhagen Psychosocial Questionnaire (CoPsoQ), desarrollado por el Arbejdsmiljpinstitittet (Instituto Nacional de Salud Laboral) de Dinamarca, el cual releva aspectos vinculados a los riesgos psicosociales en el trabajo. Para la elaboración de nuestro instrumento, tomamos las dimensiones y categorías referidas a las exigencias psicológicas cualitativas que se refieren a la invocación de emociones y sentimientos en el trabajo y a las cuantitativas, relativas a la cantidad de tarea. Los módulos que tratan sobre condiciones de trabajo, contenido y exigencias fueron adaptados a las características de la población a relevar.
- Adaptación del cuestionario ISTAS21 realizada por el Taller de Estudios Laborales ${ }^{(40)}$. Dicha fuente conformó un insumo central para la medición de los módulos de salud y condiciones laborales.

- Adaptación de la encuesta Las condiciones de trabajo y salud de los docentes privados, llevada a cabo por el Sindicato Argentino de Docentes Privados ${ }^{(41)}$. En nuestro caso, se reelaboró el bloque de preguntas referidas a las tareas docentes y el agrado/desagrado respecto de estas, cuyo modelo fue el trabajo de Mendizábal ${ }^{(42)}$.

- Para la elaboración del bloque sobre estructuración del tiempo se consultó la Encuesta de usos del tiempo ${ }^{(43)}$ desarrollada por la Dirección General de Estadística y Censos del Gobierno de la Ciudad de Buenos Aires. Su potencial radica en medir la cantidad de tiempo que los sujetos invierten en la realización de diversas actividades, tales como el trabajo remunerado, quehaceres del hogar, cuidado de personas, tiempo de ocio y entretenimiento, trabajo voluntario y tiempo destinado a traslado, entre otros. Nos interesó determinar la evaluación de la suficiencia/insuficiencia de los tiempos en relación con diversas actividades. En este sentido, indagamos cómo las condiciones de trabajo influyen sobre el trabajador fuera de su tiempo laboral, la percepción de ese tiempo con relación a cuestiones vinculares, familiares, recreativas y de salud.

- Para la medición de las formas de participación e interacción elaboramos un bloque de preguntas a partir de la lectura y sistematización de bibliografía especializada en el tema ${ }^{(44,45,46)}$. Es pertinente aclarar que estas dimensiones, generalmente, no son abordadas en estudios sobre condiciones de trabajo, ya que la perspectiva analítica se centra en el individuo.

Como parte de la estrategia participativa, se realizaron talleres de sensibilización y discusión con docentes y delegados a fin de integrar la perspectiva de los sujetos en la construcción de las dimensiones bajo análisis.

El cuestionario consta de cinco módulos temáticos, principalmente constituidos por 
preguntas cerradas: datos personales y laborales (situación personal, de formación y laboral, características demográficas de la población censada); salud (presencia de molestias o problemas de salud que estén relacionados con su labor); condiciones laborales (exigencias del puesto en función de los tiempos, las tareas, los recursos institucionales y materiales, la toma de decisiones y resolución de problemas, y los aspectos emocionales, creativos y cognitivos del trabajo); interacciones y participación (relaciones entre los sujetos que conforman la comunidad educativa, formas de participación y percepción del reconocimiento de las tareas); estructuración del tiempo (distribución entre tiempo de trabajo y no laboral).

Con el propósito de verificar la adecuación del cuestionario a los objetivos propuestos, el grado de comprensión de las preguntas y detectar incoherencias o falencias, se realizó una prueba piloto con diez docentes de diferentes unidades académicas y tipos de cargos. Posteriormente, se evaluaron los resultados y se propusieron ajustes y modificaciones.

Con el fin de asesorar a los docentes, se realizaron talleres informativos. El cuestionario fue anónimo y autoadministrado, es decir, cada docente respondió individual y voluntariamente por la actividad que desarroIlaba en la unidad académica censada. En este proceso fue relevante la participación de los delegados sindicales en el reparto y recupero de cuestionarios y el establecimiento de vías de comunicación con el plantel docente. La devolución del material se realizó a través de una urna dispuesta para el depósito de los cuestionarios. El relevamiento se llevó a cabo en 2013 y tuvo una duración de un mes.

El porcentaje de no respuesta se debió en mayor medida a ausencias o licencias durante el momento de relevamiento. Por otro lado, en la valoración del cuestionario, el bloque de preguntas sobre la identificación del cargo fue el que admitió mayor cantidad de no respuesta debido a la posibilidad de asociación simple entre la persona y su cargo.

Finalmente se realizó un análisis estadístico-descriptivo multivariado, procesado a través de tablas dinámicas de Excel y del Statistical Package for the Social Sciences (SPSS).

La realización de esta investigación fue acordada por convenio paritario entre las autoridades de la Universidad Nacional de Cuyo y los representantes de la Asociación de Docentes e Investigadores de la Universidad Nacional de Cuyo (FADIUNC). En este acuerdo intervinieron miembros de la comisión de Higiene y Seguridad, Calidad y Medio Ambiente del Trabajo de la UNCuyo y del Sindicato de Docentes Universitarios (FADIUNC), el cual fue aprobado por el Ministerio de Trabajo, Empleo y Seguridad Social de la Nación ${ }^{[a]}$.

\section{PRESENTACIÓN DE LOS RESULTADOS}

\section{Características de la población docente estudiada}

La caracterización de la población de estudio según variables sociodemográficas básicas (sexo, edad, estado civil y nivel de instrucción) es central para comprender la incidencia de las condiciones y la situación laboral de los docentes. En el estudio realizado, la distribución por sexo en la unidad académica universitaria presentó un $48 \%$ de mujeres y $52 \%$ de varones; en tanto, en la unidad académica de enseñanza media la mayoría de los docentes eran mujeres $(79 \%$ del total).

Con respecto al rango de edad, los docentes de la universidad tenían entre 27 y 67 años, con un promedio de 49 años, y el $40 \%$ de este plantel poseía más de 55 años de edad; mientras que, en los de enseñanza media, el promedio era de 44 años y la distribución de las edades del $71 \%$ del plantel docente se concentraba entre los 36 y 55 años de edad.

Según estado civil, el $64 \%$ de los censados en la universidad y el $49 \%$ en enseñanza media, estaban casados. Considerando la cantidad de hijos se verifica que, en general, no poseían más de dos ( $73 \%$ en la universidad y el $76 \%$ en enseñanza media). 
En cuanto al nivel de escolarización alcanzado, el $29 \%$ de los docentes de enseñanza media superaba el nivel universitario completo, mientras que un $79 \%$ de los docentes universitarios contaban con títulos de posgrado (especializaciones, maestrías y/o doctorados).

Respecto al tipo de cargo, en la unidad académica universitaria existían 142 cargos docentes, el $18 \%$ eran titulares y el $19 \%$ adjuntos, y la mayoría de los docentes presentaba cargos de menor jerarquía: el 31\% jefes de trabajos prácticos y el $26 \%$ ayudantes de primera. Por otro lado, en cuanto a la dedicación docente, un $24 \%$ detentaba dedicación exclusiva, un $24 \%$ semiexclusiva y el $52 \%$ dedicación simple. Una amplia porción de los docentes $(70 \%)$ mencionó que realizaba labores de investigación.

En cuanto a los docentes de enseñanza media, según tipo de contrato, el $41 \%$ contaba con un cargo titular efectivo, el 50\% con una suplencia por cargo vacante y el $9 \%$ con una suplencia por tiempo determinado.

Con respecto a la antigüedad en el cargo, el promedio de años en la universidad era de 15,3 años, en tanto que, en enseñanza media, era de 17 años.

Los datos anteriores se completan con los registros de ausentismo y de "días caídos"[b], elaborados por la universidad para el período de referencia ${ }^{(47)}$. Durante el año 2012 y el primer semestre de 2013, se otorgaron 108 licencias en total que justificaban 797 días caídos para los docentes de la universidad, en tanto que, en la unidad de enseñanza media, se verificaron 664 licencias y 3.507 días caídos.

Con respecto a las causas de las licencias, los docentes universitarios esgrimieron: motivos psiquiátricos (30\%, 240 días), intervenciones quirúrgicas ( $23 \%, 187$ días), problemas de articulaciones (13\%, 106 días), inconvenientes cardiovasculares ( $6 \%, 47$ días), cuidado de algún familiar (5\%, 43 días) u otros motivos (23\%, 174 días); mientras que los docentes de enseñanza media mencionaron: motivos psiquiátricos $(27 \%, 955$ días), licencia por maternidad $(20 \%, 698$ días), problemas vinculados con embarazos (14\%, 499 días), cuidado de algún familiar (7\%, 254 días), problemas osteoarticulares $(7 \%, 247$ días), enfermedades infecciosas (4\%, 156 días), cirugías (3\%, 123 días), por problemas respiratorios $(2 \%, 77$ días) y otros motivos (15\%, 517 días).

En síntesis, la población considerada se encuentra en tramos etarios centrales, detenta un alto nivel de posgraduación en relación con su escolarización, fundamentalmente entre los docentes de la unidad académica universitaria (un $79 \%$ respecto a la totalidad de los docentes censados) y la mitad presenta cierta estabilidad en su cargo. La distribución por edad repercute en otras dimensiones consideradas como la antigüedad en el puesto o las licencias registradas (cantidad y tipo).

\section{Salud laboral: el "cuerpo olvidado"}

Según los expertos en psicopatología del trabajo, es necesario evaluar la salud laboral como proceso complejo de construcción social, en sus diversos aspectos físicos, psíquicos y emocionales. Las dimensiones que formaron parte del módulo de salud física fueron: molestias en la voz/cuerdas vocales; dolor en los brazos/braquialgia; dolor cer$\mathrm{vical/cervicalgia,} \mathrm{dolor} \mathrm{lumbar/lumbalgia,}$ dolor de cabeza, dolor de espalda, dolor de piernas, dolor de pecho (pectoral), molestias gastrointestinales, manifestación alérgica y otros problemas.

El 42,5\% de los censados manifestó tener "algunas veces" $\mathrm{y}$ "muchas veces" problemas en la voz y en las cuerdas vocales mientras que, el $50 \%$, dolores cervicales y el $37,4 \%$ expresó padecer lumbalgias. Esta patología, junto con dolores cervicales, se encuentran asociadas a posturas sedentes propias de esta actividad laboral. Por otro lado, más del $44 \%$ de los docentes declaró padecer "algunas veces" y "muchas veces" dolores de cabeza.

Los docentes fueron consultados acerca de la asociación entre su malestar físico y las condiciones de trabajo $y$, de modo significativo, la mayoría (más de un 50\%) vinculó las molestias o dolores mencionados, en general, al desempeño de sus tareas; mientras 
que, específicamente, el $80 \%$ asoció las molestias en la voz al trabajo y el $75 \%$ asoció la cervicalgia a su actividad.

Sin embargo, Ilama la atención que los docentes no reconocieron mayoritariamente ausentarse por las molestias o los dolores provocados por la práctica profesional. Solo el $17 \%$ asumió el ausentismo por molestias en la voz, mientras el dolor cervical fue reconocido como causa de ausentismo únicamente por el $10 \%$.

Es destacable que los docentes relativizaran la asociación entre carga física del trabajo, enfermedades y ausentismo, mientras que el vínculo entre los componentes emocionales de la salud y el trabajo son ampliamente reconocidos. En este sentido, la mayoría de los docentes relacionaron el agotamiento, nerviosismo e irritabilidad como expresiones del malestar psíquico asociado al trabajo. La desconcentración, el desgano y la preocupación constante como cargas emocionales ligadas al ámbito laboral, superaron el $30 \%$ de los casos (Cuadro 1).

El conjunto de las dimensiones indagadas expresan diferentes síntomas ligados al estrés, principalmente aquellos referidos a conductas que se ponen de manifiesto mediante el nerviosismo, la irritabilidad o hábitos cambiantes. Estos se evidencian en la dificultad para conciliar el sueño, la conducta emocional variable, la angustia, el miedo, el desinterés, la preocupación constante y síntomas fisiológicos como la sudoración, palpitaciones y mareos. Considerando los datos anteriores, podemos mencionar que solo el $30 \%$ del plantel censado no presenta al menos un síntoma de estrés.

La información sobre malestar psicosocial fue recabada en un bloque de preguntas acerca del consumo de medicamentos (Cuadro 2). El 18,6\% de los docentes manifestó que tomaba tranquilizantes, antidepresivos y ansiolíticos. Además, un 16\% admitió que realizaba tratamiento psicoterapéutico, aunque no vinculaban este aspecto al trabajo. Otras medicaciones mencionadas fueron: relajantes musculares $(22,8 \%)$, antiácidos/ antiespasmódicos $(28 \%)$ y para la presión arterial y el corazón (18\%). Las patologías señaladas de tipo gástricas, cardíacas y musculoesqueléticas están vinculadas al padecimiento de estrés laboral ${ }^{(25)}$.

En resumidas cuentas, los docentes asocian los problemas emocionales y psíquicos a sus tareas y, en menor medida, los relativos al aspecto físico. Observamos un importante porcentaje que autoadministra medicamentos y el desgaste integral de su salud.

\section{Condiciones laborales: la falta de reconocimiento}

Comprendemos el esfuerzo que despliegan los trabajadores como "intensidad" del trabajo que puede desglosarse en dos aspectos: carga mental y carga psíquica. La primera refiere a la captación de señales e informaciones, su procesamiento, utilización de la memoria, resolución de problemas, adopción de decisiones y evaluación de actividades, entre las más importantes. La segunda alude a la iniciativa, obtención de resultados, estatus social de la actividad, grado de comunicación, cooperación, relaciones con otros y nivel de responsabilidad ${ }^{(48)}$. Ambos

Cuadro 1. Malestares psíquicos y físicos asociados con el trabajo reportados por docentes universitarios y de enseñanza media. Porcentajes según tipo de malestar (n=193). Mendoza, Argentina, 2013.

$\begin{array}{lrrr}\text { Malestares psíquicos y físicos } & \text { Sí } & \text { No } & \begin{array}{c}\text { Sin } \\ \text { dato }\end{array} \\ \text { Agotamiento } & 59,6 & 36,8 & 3,6 \\ \text { Nerviosismo o tensión } & 56,0 & 39,9 & 4,1 \\ \text { Desconcentración o problemas para concentrarse } & 35,8 & 59,1 & 5,1 \\ \text { Desgano o desmotivación } & 36,3 & 57,5 & 6,2 \\ \text { Preocupación constante } & 36,3 & 57,0 & 6,7 \\ \text { Irritabilidad o mal humor } & 35,2 & 59,1 & 5,7 \\ \text { Dificultades para conciliar el sueño } & 23,8 & 70,5 & 5,7 \\ \text { Sudoración y/o palpitaciones } & 11,9 & 81,3 & 6,7 \\ \text { Angustia o miedo } & 12,4 & 80,3 & 7,3 \\ \text { Mareos u obnubilaciones } & 9,8 & 82,9 & 7,3 \\ & & & \\ \text { Fuente: Elaboración propia sobre la base del Primer Censo de Condiciones y } & \text { Salud } \\ \text { Laboral. } & & & \end{array}$


Cuadro 2. Consumo de medicamentos reportado por docentes universitarios y de enseñanza media. Porcentajes según tipo de medicación (n=193). Mendoza, Argentina, 2013.

$\begin{array}{lccc}\text { Tipo de medicación } & \text { Sí } & \text { No } & \begin{array}{c}\text { Sin } \\ \text { dato }\end{array} \\ \text { Calmantes del dolor (analgésicos) } & 37,8 & 58,0 & 4,1 \\ \text { Relajantes musculares (para contracturas o dolores musculares) } & 22,8 & 72,5 & 4,7 \\ \text { Psicofármacos (tranquilizantes, antidepresivos, ansiolíticos, etc.) } & 18,6 & 76,7 & 4,7 \\ \text { Medicación para el aparato digestivo (antiácidos, antiespasmódicos, etc.) } & 28,0 & 67,9 & 4,1 \\ \text { Medicación para el corazón, problemas cardíacos, presión arterial, etc. } & 18,1 & 76,7 & 5,2 \\ \text { Antialérgicos } & 26,4 & 68,4 & 5,2\end{array}$

aspectos pueden potenciar sus impactos en la vida del trabajador cuando se dan situaciones laborales en las cuales la organización del trabajo es altamente jerarquizada y los trabajadores se encuentran sujetos a un importante control en relación con las formas de hacer, ser y estar en el trabajo. Sin embargo, el control en exceso o las cargas laborales inadecuadas pueden no ser percibidas de este modo por los trabajadores o pueden encontrarse "naturalizadas"(49,50). Así, es necesario contrastar estos aspectos con dos dimensiones relacionadas: la satisfacción en el trabajo y la implicación que el trabajador construye hacia este. La satisfacción laboral puede concebirse como "el grado en que una persona experimenta sentimientos positivos o negativos en relación con los diversos aspectos del trabajo"(51), mientras que la implicación con el trabajo está compuesta por tres aspectos: la identificación psicológica hacia el trabajo, las contingencias desempeño-autoestima, y los sentimientos de deber y obligación hacia el trabajo, según el estudio clásico de Lodah y Kejner ${ }^{(52)}$.

La intensidad del trabajo (en este caso, captada bajo la dimensión de carga mental) se analizó a través de la rapidez en la realización de las tareas, su acumulación y los descansos pautados. En una primera aproximación, los docentes declararon llevar al día su trabajo en una muy alta proporción (entre siempre y frecuentemente $98,5 \%$ ). Sin embargo, aceptaban que sus tareas denotaban ciclos de variación en sus cargas. De este modo, un 58\% expresó que su carga laboral "raras veces" o "nunca" disminuía, mientras que un 80,8\% reconoció acumulaciones de trabajo "siempre" o "frecuentemente" (Cuadro 3).

La cuestión de la rapidez se relativiza en estas actividades (docentes y de apoyo de enseñanza) ya que, generalmente, se vincula a la velocidad motriz. No obstante, un $73 \%$ del plantel manifestó que "siempre" o "frecuentemente" tenía que realizar su actividad de este modo.

En referencia a la carga psíquica del trabajo, un 53\% reconoció que le costaba olvidar "frecuentemente" los problemas laborales $y$, casi un $50 \%$, que el trabajo los desgastaba emocionalmente. Si sumamos a quien aceptó que la situación de desgaste se producía "raras veces" tenemos que, en algún momento del año y del desempeño laboral, el $75 \%$ sintió desgaste emocional. Desde otra arista, los docentes declararon que su trabajo requería de la toma constante de decisiones (el $89,6 \%$ entre "siempre" y "frecuentemente") y que debían controlar tareas en simultáneo (el $85,5 \%$ entre "siempre" y "frecuentemente"). 
La correspondencia entre el trabajo y los valores personales evidenció una vinculación alta: el $63 \%$ de los docentes aceptaron que "nunca" el trabajo los puso en contradicción con sus valores.

\section{Autonomía e influencia en el trabajo: roles y sentidos}

La influencia y la autonomía en el trabajo refieren a la toma de decisiones en la realización de las tareas y la posibilidad de expresar habilidades y conocimientos. También se vincula con la suficiencia de los tiempos de descanso y el sentido "en" y "por" el trabajo, el compromiso con la profesión (implicación) y la percepción entusiasta. La escasez de influencia o la limitación de la autonomía exponen a los trabajadores a un sentimiento de exigencia y autoexigencia crecientes. El control sobre los tiempos de trabajo se refiere a la capacidad para decidir sobre los ritmos (pausas, trámites, permisos). La falta de control y el autocontrol sobre estos se convierten en un aspecto que afecta el bienestar de los trabajadores ${ }^{(53)}$.
En cuanto al control sobre la cantidad de trabajo asignada, el 18,1\% manifestó que "nunca" tuvo influencia sobre dicha cantidad y un $45 \%$ que solo "alguna/s veces". A esto se añade un alto porcentaje $(69,4 \%)$ que recibió exigencias contradictorias "siempre" o "alguna vez" en realización con su labor. Solo el 32,6\% de los docentes percibió que la cantidad de alumnos "nunca" excedió su capacidad laboral (el 66,4\% aceptó que los excedía en "alguna" medida).

Es relevante destacar las respuestas que se obtuvieron sobre las "imposiciones" en el trabajo: una importante porción fue categórica al expresar que el trabajo "nunca" excedía su formación (52,3\%); "nunca" se le imponían tareas que no les competían (55\%); "nunca" hacían tareas que deberían hacer otros $(40,4 \%)$ y "nunca" les exigían tareas innecesarias $(47 \%)$. Sin embargo, un tercio de los trabajadores denotó que "alguna/s veces" en el último año sintió que el trabajo excedía su puesto (38,9\%); que le impusieron tareas que no les competían al menos "una vez" $(35,7 \%)$; que debieron hacer tareas de otros $(49,7 \%)$; que les exigieron tareas innecesarias $(46 \%)$.

Cuadro 3. Percepción de la intensidad de trabajo en docentes universitarios y de enseñanza media. Porcentajes según aspectos y frecuencia (n=193). Mendoza, Argentina, 2013.

\begin{tabular}{|c|c|c|c|c|c|}
\hline Intensidad de trabajo & Siempre & Frecuentemente & $\begin{array}{l}\text { Raras } \\
\text { veces }\end{array}$ & Nunca & Sin dato \\
\hline ¿Tiene que trabajar rápido? & 10,9 & 62,2 & 14.4 & 9,3 & 3,6 \\
\hline ¿Hay periodos en que se le acumula trabajo? & 10,4 & 70,4 & 9,8 & 8,8 & 0,6 \\
\hline $\begin{array}{l}\text { ¿Hay periodos en que disminuye considerablemente } \\
\text { su carga de trabajo? }\end{array}$ & 1,6 & 38,3 & 35.8 & 22,3 & 2,1 \\
\hline $\begin{array}{l}\text { ¿Para usted son suficientes los tiempos } \\
\text { de descanso pautados? }\end{array}$ & 20,2 & 51,8 & 17,6 & 8,3 & 2,1 \\
\hline ¿Lleva al día su trabajo? & 41,5 & 57,0 & 0,5 & 0.0 & 1.0 \\
\hline ¿Le cuesta olvidar los problemas del trabajo? & 11,4 & 52,9 & 16,6 & 16,6 & 2,5 \\
\hline ¿Su trabajo es desgastante emocionalmente? & 5,7 & 49,7 & 25,9 & 17,1 & 1,6 \\
\hline ¿Usted puede controlar sus emociones en el trabajo? & 40,9 & 52,4 & 2,6 & 2,6 & 1,5 \\
\hline ¿Su trabajo entra en contradicción con sus valores? & 1,6 & 13,5 & 19,7 & 63,2 & 2,0 \\
\hline ¿Su trabajo requiere que tome decisiones constantemente? & 30,6 & 59,0 & 5,2 & 3.6 & 1,6 \\
\hline $\begin{array}{l}\text { ¿Su trabajo requiere controlar diversas tareas } \\
\text { en forma simultánea? }\end{array}$ & 32,6 & 52,9 & 8,3 & 5,2 & 1,0 \\
\hline
\end{tabular}


Sobre el sentido del trabajo, se destaca que casi la totalidad (97\%) de los docentes se sentía comprometido con su profesión; el $87 \%$ se expresaba ("siempre" y "muchas veces") con entusiasmo sobre su trabajo frente otros, un $78 \%$ percibía que contaba con los recursos materiales necesarios para desarrollar su trabajo y, un $74,6 \%$, con recursos institucionales.

La síntesis de los datos nos indica que los trabajadores docentes tenían una percepción positiva sobre los medios para realizar su trabajo y sobre su propia capacidad para desplegar sus tareas, a la vez que reconocían un alto compromiso con su realización, aunque la distribución de esas tareas se percibía como desigual para un tercio del plantel censado.

\section{Organización laboral: mando y grupo}

Las características de mando refieren a las formas de gestión jerárquicas de cada organización, las cuales ponen de manifiesto las relaciones docentes-autoridades. En este marco se entiende por previsibilidad el hecho de disponer de la información adecuada, suficiente y a tiempo para adaptarse a los cambios organizacionales, tecnológicos, de la tarea, metodológicos, etc., mientras que la baja previsibilidad supone una situación de riesgo e incertidumbre que puede perjudicar a los trabajadores.

Un alto porcentaje de docentes sabían cuáles eran las tareas bajo su responsabilidad (96\% "siempre" y "muchas veces") y contaban con la información necesaria para realizar su trabajo (92\% "siempre" y "muchas veces"). Por otra parte, percibió un importante apoyo institucional: un $72,5 \%$ advirtió que los directivos planificaban en tiempo y forma el trabajo y, en menor grado, que se comunicaban de manera fluida $(62,7 \%$ "siempre" $y$ "muchas veces").

El sentimiento de grupo refiere a la calidad de la relación con los compañeros de trabajo y la posibilidad de formar parte de un equipo. Este sentido de pertenencia fomenta relaciones de solidaridad y compañerismo. En este ítem el 83,4\% advirtió que recibía ayuda de sus compañeros; el 75\% sintió que formaba parte de un grupo, mientras que el porcentaje disminuye a un $59,1 \%$ en referencia a la realización de tareas o proyectos en equipo.

La información obtenida en este bloque denota que existe una importante verticalidad en la organización del trabajo sumada a una fuerte identificación de tipo corporativa, que no necesariamente se traslada a la realización grupal de tareas.

\section{Tareas y grado de satisfacción}

Este apartado indica la relación entre las diversas actividades realizadas por los docentes, la cantidad de personal que efectivamente las ejecuta y el grado de satisfacción que su realización produce. De los datos obtenidos se desprende que la mayoría de los docentes eran polifuncionales debido a los diferentes tipos de tareas que asumían y asociaban: además de las propiamente docentes (94\%), el $73 \%$ daba contención y apoyo a sus alumnos; el $43 \%$ hacía investigación; el $28 \%$ tareas de extensión; el 30\% tareas administrativas; el $42 \%$ pedagógicas; el $70 \%$ asistía a reuniones institucionales.

A esta carga hay que sumar las de formación que fueron indicadas al principio y evaluarlas con relación al alto porcentaje de docentes que declaró tener sus tareas al día. También es importante destacar que la investigación y extensión generalmente traen asociadas (para los docentes a cargo de la dirección de proyectos), actividades de organización y administración de recursos financieros. La mayoría de las actividades desempeñadas demuestra un alto grado de satisfacción para los docentes censados, principalmente aquellas relacionadas directamente con su profesión.

\section{Inseguridad en el trabajo}

Esta categoría refiere a la temporalidad y precariedad laboral, vinculadas a la relación salarial y contractual (duración, términos y condiciones). El Cuadro 4 muestra dos 
Cuadro 4. Preocupación laboral reportada por docentes universitarios y de enseñanza media. Porcentajes según aspectos y grado de preocupación (n=193). Mendoza, Argentina, 2013.

\begin{tabular}{|c|c|c|c|c|c|}
\hline Aspectos & $\begin{array}{c}\text { Muy } \\
\text { Preocupado }\end{array}$ & $\begin{array}{c}\text { Algo } \\
\text { preocupado }\end{array}$ & $\begin{array}{c}\text { Poco } \\
\text { preocupado }\end{array}$ & $\begin{array}{c}\text { Nada } \\
\text { preocupado }\end{array}$ & Sin dato \\
\hline Obtener más horas/ turnos & 6,2 & 26,4 & 21,2 & 44,6 & 1,6 \\
\hline Reducción de horas/ turnos & 6,7 & 16,1 & 18,1 & 54,9 & 4,2 \\
\hline Cambios de horario (turno, horas, etc.) & 5,2 & 19,7 & 19,2 & 52,3 & 3,6 \\
\hline Salario & 27,5 & 51,3 & 9,3 & 10,4 & 1,6 \\
\hline Continuidad de la relación laboral & 15,5 & 24,4 & 19,2 & 38,9 & 2,1 \\
\hline Otros & 4,2 & 5,2 & 1,6 & 44,0 & 45,1 \\
\hline
\end{tabular}

características básicas: en primer lugar, la estabilidad laboral de una porción importante de la población y, en segundo lugar, que la variable de ajuste de la relación laboral la conforma el salario. En este sentido, las preocupaciones de los trabajadores se centraron en el nivel salarial (el $79 \%$ entre "muy preocupados" y "algo"), mientras que en un $40 \%$ la preocupación era la continuidad del vínculo laboral ("muy preocupado" y "algo preocupado").

La mayoría de los censados mencionó estar "nada preocupado" por obtener más horas o turnos de trabajo $(44,6 \%)$, por la reducción de horas $(55 \%)$ o por sufrir cambios de horarios (52,3\%). Esto demuestra tanto la sobrecarga laboral como la estabilidad horaria entre el plantel.

Es importante destacar que una porción altamente importante de los censados mostraron preocupación por otros motivos tales como: aumento de la dedicación, régimen de jubilación y el desdoblamiento de cargas de trabajo.

\section{Interacciones y participación: la individualización}

La información registrada aquí pretende captar dimensiones que comprendan al trabajo en su dimensión colectiva y organizacional. Los riesgos psicosociales y organizacionales dependen de las formas en que se organiza el trabajo y de las relaciones que se entablan entre pares, con los superiores, con la organización sindical y con los alumnos y sus familiares. Por ello, este bloque da cuenta de la valoración de los docentes sobre estos actores, la presencia de agresiones o persecuciones y cuáles consideran que son los principales problemas que presenta la unidad académica, los canales de resolución de demandas y el reconocimiento del desempeño en el trabajo. Estas dimensiones son aspectos fundamentales que hacen referencia a la categoría estima en el trabajo como una forma de reconocimiento, apoyo adecuado y trato justo.

Por otro lado, pretendimos captar las formas de participación de los docentes, la incidencia del sindicato en las condiciones de trabajo, los niveles de afiliación y el conocimiento de las principales normativas que regulan sus actividades. Estos aspectos son centrales para evidenciar las formas de canalización, organización y resolución de los problemas laborales, es decir, comprender los modos de gestión individual o colectiva de las situaciones conflictivas del trabajo.

Del análisis del Cuadro 5 y del Cuadro 6 es posible indicar los siguientes aspectos: alrededor del $70 \%$ de los docentes declaró mantener una relación "excelente" y "muy buena" con el personal jerárquico, en sus espacios de trabajo. Sin embargo, más del $50 \%$ 
Cuadro 5. Valoración de las relaciones en docentes, universitarios y de enseñanza media. Porcentajes según los actores y la calidad de la relación (n=193). Mendoza, Argentina, 2013.

\begin{tabular}{|c|c|c|c|c|c|c|}
\hline Valoración de la relación con... & Excelente & $\begin{array}{l}\text { Muy buena } \\
\text { / Buena }\end{array}$ & Regular & Mala & $\begin{array}{l}\text { Sin } \\
\text { relación }\end{array}$ & $\begin{array}{l}\text { Sin datos / No } \\
\text { corresponde }\end{array}$ \\
\hline $\begin{array}{l}\text { los directivos o jerárquicos en el ámbito } \\
\text { de trabajo }\end{array}$ & 29,5 & 57,5 & 4,1 & 1,0 & 4,1 & 3,6 \\
\hline el personal jerárquico de la UNCuyo & 8,3 & 40,4 & 3,6 & 0,5 & 38,3 & 8,8 \\
\hline el inmediato superior & 43,0 & 49,3 & 5,2 & 0,5 & 1,0 & 1,0 \\
\hline otros colegas/docentes & 31,6 & 66,8 & 0,5 & 0,0 & 0,0 & 1,0 \\
\hline $\begin{array}{l}\text { el personal de apoyo docente sector } \\
\text { administrativo }\end{array}$ & 33,7 & 63,2 & 1,0 & 0,0 & 0,5 & 1,5 \\
\hline el personal de apoyo docente en general & 29,5 & 67,4 & 1,0 & 0,0 & 0,0 & 2,0 \\
\hline los alumnos & 39,4 & 59,1 & 0,5 & 0,0 & 0,5 & 0,5 \\
\hline
\end{tabular}

expresó que "algunas veces", "raras veces" o "nunca" recibió reconocimiento por parte de las autoridades y jerarquías. Por otro lado, una porción importante del plantel asumió ausencia de relación (más del 38\%) con las esferas más altas de la universidad.

En referencia a los vínculos entre pares, los docentes mencionaron tener excelentes $y$ muy buenas relaciones en un nivel horizontal, ya sea con docentes de la misma cátedra y con docentes de la institución en general.
Si bien mayoritariamente manifestaron sentirse reconocidos por sus colegas, también es alto el nivel de respuesta de aquellos que se sintieron reconocidos "ocasionalmente" o "nunca" $(38,7 \%)$.

En tanto la relación con los alumnos también mostró valores positivos. Casi la totalidad de las respuestas se concentró entre aquellos que expresaron que era "excelente" y "muy buena". Esta tendencia no se mantuvo en relación con el reconocimiento

Cuadro 6. Reconocimiento y apoyo percibido por docentes, universitarios y de enseñanza media, Porcentajes, según actores y frecuencia $(n=193)$. Mendoza, Argentina, 2013

$\begin{array}{lcccrr}\text { Reconocimiento/apoyo de... } & \text { Siempre } & \begin{array}{c}\text { Muchas } \\ \text { veces }\end{array} & \begin{array}{c}\text { Algunas } \\ \text { veces }\end{array} & \begin{array}{c}\text { Raras } \\ \text { veces }\end{array} & \text { Nunca } \\ \text { Autoridades/jerarquías } & 14,3 & 33,9 & 30,2 & 15,3 & 6,3 \\ \text { Colegas y pares } & 14,7 & 46,6 & 29,3 & 6,3 & 3,1 \\ \text { Sindicato } & 1,2 & 5,4 & 11,3 & 16,1 & 66,1 \\ \text { Alumnos } & 16,0 & 46,5 & 23,0 & 8,0 & 6,4 \\ \text { Padres/familiares de los alumnos } & 4,1 & 13,0 & 17,8 & 15,4 & 49,7 \\ \text { Fuente: Elaboración propia sobre la base del Primer Censo de Condiciones y Salud Laboral. } & & \end{array}$


del desempeño por parte del alumnado, el $37,4 \%$ sostuvo que eran reconocidos "algunas veces", "raras veces" o "nunca".

La relación con los padres o familiares de los alumnos se caracterizó por ser nula o por no corresponder (en el caso del ámbito propiamente universitario), por lo cual, son bajos los niveles de reconocimiento.

La relación con la organización sindical es altamente relevante para analizar la posibilidad de defensa de los intereses del colectivo laboral(54,55). Casi un tercio de los docentes la valoró como "excelente" y "muy buena"; sin embargo, un $37,8 \%$ manifestó no tener relación con la organización. Esta situación también se evidencia en que más del $65 \%$ de los censados que expresaron no sentirse "nunca" reconocidos por el sindicato.

En relación con las agresiones en el lugar de trabajo (física, verbal, psicológica, hostigamiento o acoso sexual), el $77 \%$ de los censados respondió no haber sufrido ninguna de ellas. En tanto, el 23\% restante expresó el padecimiento de al menos una de las agresiones mencionadas. El análisis de aquellos que efectivamente sufrieron algún tipo de agresión indicó que el $41 \%$ fue ejercida por directivos o personal jerárquico, principalmente agresiones verbales y psicológicas; un $5 \%$ fueron ejercidas por compañeros de trabajo docentes y un $8 \%$ por compañeros no docentes; un $38 \%$ proviene de los alumnos, ya sea psicológica o verbal; $y$, finalmente un $8 \%$ fueron efectuadas por los familiares de los alumnos.

Uno de los aspectos que se indagó a través de preguntas abiertas buscó identificar los principales problemas laborales. Aquí es necesario realizar una distinción entre unidades académicas. En la universidad los docentes resaltaron mayoritariamente la falta de cargos y la dedicación simple de los cargos en ejercicio; en tanto los docentes de enseñanza media plantearon mayoritariamente los problemas de comunicación dentro del establecimiento y la falta de titularización. Asimismo indicaron como una debilidad la falta de espacio físico, de personal y el hacinamiento áulico. También expresaron la sobrecarga de tareas que padecen en sus labores cotidianas y las dificultades que acarrea el uso de la tecnología (celulares, netbooks) por parte de los alumnos. Es decir, aparece un cuestionamiento a la relación docentealumno que marcaría al menos un contraste con la declarada "excelente" o "muy buena" vinculación entre ambos.

Con respecto a los canales a los que acuden los docentes para resolver problemas laborales se estableció que, en primer lugar $(70 \%)$ se acude al jefe o superior de la organización laboral, en segundo lugar (64\%) se apela a los compañeros de trabajo ( $24 \%$ ), en tercer lugar al delegado sindical y por último, al sindicato (8\%).

Finalmente, los docentes manifestaron una alta participación en reuniones laborales $(83,3 \%)$ y en medidas de acción directa $(76,3 \%)$. Sin embargo, esta elevada participación presentó la tendencia contraria si se observan los valores de participación en reuniones sindicales dentro y fuera del lugar de trabajo o en el local sindical. Solo el $4 \%$ del plantel docente participaba de instancias gremiales por fuera del ámbito de trabajo. Estos bajos niveles, sumados a la recurrencia al personal jerárquico como vía de resolución de sus problemáticas, implicarían una asunción predominantemente individual en la gestión de los conflictos. Pese a ello, los docentes mantienen altos niveles en materia de acción directa, lo cual no es contradictorio sino que estaría indicando la carencia de instancias de mediación.

Si bien en ambas instituciones los niveles de afiliación sindical eran altos con relación a la media nacional del $39,7 \%{ }^{(56)}$, los docentes de la universidad presentaron valores cercanos al $60 \%$, en tanto que los de enseñanza media a un $50 \%$. Mayoritariamente, los docentes consideraron como "altamente positiva" (16\%) y "positiva" $(61,4 \%)$ la existencia del sindicato en la incidencia de sus condiciones laborales, sin embargo -como se mencionó- se recurre al sindicato como la última opción para la resolución de los problemas laborales. La indiferencia en relación con su presencia alcanzó el $22 \%$. 


\section{Estructuración del tiempo: la doble presencia}

La relevancia de evaluar la distribución del tiempo (laboral y extralaboral) radica en la necesidad de identificar las exigencias que impone a cada trabajador el tiempo productivo sobre los tiempos reproductivos y de expansión individual. Por ello este módulo se centra en el uso del tiempo en el hogar, la realización de tareas domésticas, el ocio y el tiempo libre.

El $40 \%$ de los docentes realizaban la mayor parte de las tareas familiares y domésticas como "el principal responsable". De este total, el $95 \%$ eran mujeres, mientras que de aquellos que "solo hacen tareas muy puntuales" ( $12 \%$ del total), el $79 \%$ eran varones.

De las docentes mujeres, el $56 \%$ era la principal responsable de las tareas domésticas y familiares, mientras que el $38 \%$ de los varones reconoció realizar una cuarta parte y el $31 \%$ solo tareas puntuales.

Las mujeres trabajadoras se responsabilizan y realizan la mayor parte del trabajo familiar y doméstico, con lo que efectúan un mayor esfuerzo de trabajo total en comparación con los hombres. Esta "doble jornada" laboral de la mayoría de mujeres trabajadoras es en realidad una "doble presencia", pues las exigencias de ambos trabajos (el productivo y el familiar y doméstico) son asumidas cotidianamente de manera sincrónica (ambas exigencias coexisten de forma simultánea). ${ }^{(25}$ p.31-32) [c]

Durante los días de semana, las actividades extralaborales de mayor peso eran las relacionadas a la reproducción y vida familiar. Se destaca la escasa importancia de actividades físicas y de cuidado personal, ocio y recreación. Los docentes reconocieron no tener tiempo o tenerlo de manera "insuficiente" en más del $60 \%$ para cualquiera de las actividades mencionadas.

Solo el $20 \%$ de los docentes valoró la disponibilidad de tiempo para dedicarse a la familia como "muy suficiente" durante los fines de semana. Entre el $10 \%$ y el $12 \%$ evaluó no contar con tiempo para ocio y tiempo libre, actividades artísticas y culturales. A lo cual se suma que el $46 \%$ percibía como "insufi-

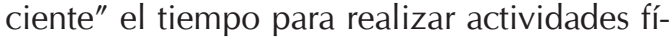
sicas y deportivas. El porcentaje de aquellos que evaluaron como "insuficiente" el tiempo para la atención personal y de salud durante los fines de semana ascendía al 30\% (60\% de los cuales eran docentes de enseñanza media). Es importante recordar aquí que la mayoría de los docentes destacaron que llevan su trabajo al día, lo que indicaría la disposición de tiempos personales para el trabajo.

\section{REFLEXIONES FINALES}

En general, las aproximaciones cuantitativas homogeneízan ciertas situaciones permitiendo una caracterización estilizada de tipo descriptiva de la población, a la vez que permiten la emergencia de datos que dan lugar a una reflexión de mayor profundidad. A simple vista, estos trabajadores -altamente calificados, mayoritariamente estables, ubicados en un momento vital de seguridad profesional- podrían considerarse privilegiados con respecto al conjunto de la población que vive del trabajo. Esta afirmación, que se desprende de los datos y de la autopercepción de los censados, contrasta fuertemente con otros datos: el $75 \%$ de los docentes reconoció desgaste emocional en el desempeño de su tarea y el $60 \%$ un estado de agotamiento mental, cuyas consecuencias -desconcentración, desgano y preocupación- se manifestaron en un tercio del plantel. Esta información tiene un asidero material que se expresa en la acumulación cotidiana de trabajo declarada por el $80,8 \%$ de los docentes y la escasa influencia sobre la magnitud de su labor, mencionada por el $63 \%$. A lo anterior se suma la manifiesta polifuncionalidad, la preocupación por los niveles salariales, la desigualdad en la distribución de los cargos y la incertidumbre con respecto al futuro (para los inactivos, la jubilación y para los activos, los interinatos y la estabilidad en el cargo) 
A lo mencionado es posible incorporar un aspecto de degradación simbólica de la labor docente que se sostiene en la falta de reconocimiento por parte de las autoridades, en los escasos vínculos con la comunidad, en la autopercepción del desmerecimiento de su labor.

El cuadro se complejiza con otro dato que podría parecer contradictorio: la alta satisfacción profesional y personal desbalancea todo registro de malestar laboral y subraya el importante grado de compromiso. Sin embargo, con la desvalorización material señalada y la degradación simbólica que la completa, podemos decir que la autoafirmación de la satisfacción laboral docente conforma el último renglón de distinción social y cultural que aún pone en juego el estatus docente.

Frente a todas estas situaciones, las resoluciones de los conflictos son de orden individual y al reenviarse a este ámbito, la salud y el propio cuerpo son los que se resienten. La cantidad de días caídos, la proliferación de intervenciones quirúrgicas y el deterioro corporal denotan un cuerpo físico escindido de su expresión emocional, configurando un síntoma que constituye uno de los datos más relevantes de la presente investigación.

A una carrera laboral que, tradicionalmente, se basa en la meritocracia individual (uno de cuyos indicadores son, sin duda, las certificaciones de posgrado), vienen a sumarse las nuevas tecnologías y dispositivos de organización, competencia y calidad laboral y evaluación personal (académica), cuyos efectos potencian los resultados advertidos. Los altos niveles detectados en términos de solvencia académica y de desempeño (los docentes no se sienten rebasados en la mayoría de los casos por el trabajo) sostienen un perfil profesional que integra una alta estima por el estatus alcanzado y una magra representación de su pertenencia a un colectivo laboral.

El conjunto de las dimensiones abordadas dejaron constancia del desgaste general que padecen los docentes, pero no de su incidencia específica: detectamos importantes asimetrías entre las unidades académicas censadas, vinculadas a la naturaleza de la labor universitaria y de la enseñanza media. Esta limitación, que se desprende de la aplicación del mismo cuestionario en los dos casos, también se expresó en la debilidad para captar las distinciones entre trabajo prescripto (el que formalmente se contrata) y trabajo real (el que efectivamente se realiza). Aun así, el instrumento empleado demostró solvencia para cumplir con los objetivos de investigación planteados.

Frente a todo lo expuesto valdría preguntarnos, desde la perspectiva social, si lo que muestran los docentes no es más que la crisis de una movilidad ascendente anclada en la vía regia de la educación. En el actual contexto, este ámbito se tornó otro campo más de igualación del trabajo por la vara de la precarización laboral. Y asociado a lo anterior, el desgaste nos muestra el punto en que se resquebraja el mito que vinculaba un mayor nivel de educación a un mejor trabajo.

\section{AGRADECIMIENTOS}

La investigación mencionada en este trabajo fue financiada mediante acuerdo paritario (30/05/2012) entre la Universidad Nacional de Cuyo y la Asociación de Docentes e Investigadores de la Universidad Nacional de Cuyo (FADIUNC) en el marco del Programa de Capacitación Gratuita en Condiciones y Medio Ambiente de Trabajo denominado: "Investigación-acción: Investigación teórica y metodológica del personal docente para la evaluación, diagnóstico y análisis de las con- diciones de trabajo del sector". Agradecemos a FADIUNC, en la persona de su secretaria general profesora Cristina Romagnolli y, especialmente, a la profesora Stella Maris Alcantú, a cargo del programa. También a los licenciados Diego Quattrini y Nicolás Musolino, miembros participantes en diferentes instancias del relevamiento. Merece una especial mención por su colaboración el cuerpo de delegados de FADIUNC (2012-2013). Especialmente queremos mencionar las observaciones oportunas realizadas por los/as evaluadores/as de la revista Salud Colectiva. 


\section{REFERENCIAS BIBLIOGRÁFICAS}

1. Antunes R, Braga R. Infoproletários: a degradação real do trabalho virtual. São Paulo: Boitempo; 2009.

2. Castillo JJ. La soledad del trabajador globalizado. Papeles de Relaciones Ecosociales y Cambio Global. 2009;(108):11-20.

3. Pacheco E, De la Garza E, Reygadas L. Trabajos atípicos y precarización del empleo. México: El Colegio de México; 2011.

4. Castel R, Kessler G, Merklen D, Murard N. Individuación, precariedad, inseguridad: ¿desinstitucionalización del presente? Buenos Aires: Paidos; 2013.

5. De la Garza E. Trabajo no clásico: organización y acción colectiva, tomo II. México: UAM-Plaza y Valdés; 2011.

6. Tenti Fanfani E. La escuela y la cuestión social. Buenos Aires: Siglo XXI Editores; 2007.

7. Mendonça Lima $M$, Oliveira Lima-Filho F. Condições de trabalho e saúde do/a professor/a universitário/a. Ciências \& Cognição. 2009;14(3):62-82.

8. Sun W, Wun H, Wan L. Occupational stress and its related factors among university teachers in China. Journal of Occupational Health. 2011;53(4):280-283.

9. Fardella C, Sisto V. Nuevas regulaciones del trabajo docente en Chile: Discurso, subjetividad y resistencia. Psicologia \& Sociedade. 2015;27(1):68-79.

10. Villagra MA. El actual trabajo docente universitario en Argentina: alertas para repensar. Educar em Revista. 2015;(57):115-129.

11. Del Bono A, Quaranta G. Convivir con la incertidumbre: aproximaciones a la flexibilización y precarización del trabajo en Argentina. Buenos Aires: CEIL-CICCUS; 2010.

12. Pérez Crespo G. Herramientas legales en la lucha sindical. En: De eso no se habla: Organización y lucha en el lugar de trabajo. Buenos Aires: Taller de Estudios Laborales; 2002.

13. Villanueva E. Empleo y globalización: La nueva cuestión social en Argentina. Buenos Aires: Universidad Nacional de Quilmes; 1997.

14. Pierbattisti D. La privatización de los cuerpos: La construcción de la proactividad neoliberal en el ámbito de las telecomunicaciones. Buenos Aires: Prometeo; 2008.

15. Plotkin M. La privatización de la educación superior y las ciencias sociales en Argentina: Un estudio de las carreras de Psicología y Economía. Buenos Aires: CLACSO; 2006.

16. Buchbinder P, Marquina M. Masividad, heterogeneidad y fragmentación: El sistema universitario argentino 1983-2008. Buenos Aires: Universidad Nacional de General Sarmiento, Biblioteca Nacional; 2008.

17. Jimenez Gamboa A. El interinazgo como indicador de precarización laboral en la universidad. Ponencia presentada en XXX Congreso Latinoamericano de Sociología ALAS, Costa Rica, 2015.

18. Groisman F, García de Fanelli A. Incentivos a la profesión académica: los salarios de los docentes universitarios en la Argentina. Revista Latinoamericana de Estudios del Trabajo. 2009;14(21):143-167.

19. Actis Di Pasquale E. La evolución salarial del docente universitario: Un análisis relativo de las remuneraciones de los cargos exclusivos 1998-2005. Faces. 2006;12(27):29-57.

20. Collado P, Emili M, Quattrini D, Soria B, Randis M. Conflictividad sindical en la Provincia de Mendoza: el protagonismo de los trabajadores del Estado. Córdoba: II Jornadas Internacionales de Problemas Latinoamericanos; 2010.

21. Araujo S. Evaluación de la actividad investigadora, incentivos y efectos en el trabajo académico: Un estudio de caso en Argentina. Revista de Educación. 2003;(331):489-506.

22. Rodríguez Izquierdo R. Repensar la relación entre las TIC y la enseñanza universitaria: Problemas y soluciones. Profesorado. 2011;15(1):10-22.

23. Dussel I, Quevedo LA. Educación y nuevas tecnologías: los desafíos pedagógicos ante el mundo digital. Buenos Aires: Santillana; 2010.

24. Neffa JC, Giraudo E, Korinfeld S, Mendizábal N, Poy M. Telegestión: Su impacto en la salud de los trabajadores. Buenos Aires: CEIL-PIETTE, FOEESITRA; 2001.

25. Moncada S, Llorens C, Kristensen T. Método ISTAS21 (CoPsoQ): Manual para la evaluación de riesgos psicosociales en el trabajo [Internet]. ISTAS; 2002 [citado 5 ago 2015]. Disponible en: http://goo.gl/5Z6Z36. 
26. World Health Organization. Official Records of the World Health Organization $N^{\circ} 2$ [Internet]. Geneva: WHO; 1948 [citado 10 dic 2015]. Disponible en: http://goo.gl/yUhhZc.

27. Maslach C. Burnout: a social psychological analysis. En: Jones JW, (ed). The burnout síndrome: current research, theory, interventions. Illions: London House Press; 1982. p. 30-53.

28. Maslach C. Burnout: The cost of caring. New York: Prentice-Hall Press; 1982.

29. Maslach C. Burnout. En: Organización Internacional del Trabajo. Enciclopedia de Salud y Seguridad en el Trabajo. Madrid: Ministerio de Trabajo y Asuntos Sociales; 2001.

30. Salanova M, Schaufeli WB, Llorens S, Peiró JM, Grau R. Desde el Burnout al engagement ¿una nueva perspectiva? Revista de Psicología del Trabajo y de las Organizaciones. 2000;16(2):117-134.

31. Gil Monte P. El síndrome de quemarse por el trabajo (burnout): Una enfermedad laboral en la sociedad del bienestar. Madrid: Pirámide; 2005.

32. Dejours C. El factor humano. Buenos Aires: Humanitas; 1998.

33. Dejours C. Trabajo y desgaste mental: Una contribución a la psicopatología del trabajo. Buenos Aires: Humanitas; 1999.

34. Baudelot C, Gollac M. ¿Trabajar para ser feliz?: La felicidad y el trabajo en Francia. CEILCONICET, Miño y Dávila Editores; 2011.

35. Hirigoyen MF. El acoso moral en el trabajo: Distinguir lo verdadero de lo falso. Barcelona: Paidós Ibérica; 2001.

36. Piñuel I. Mobbing: Cómo sobrevivir al acoso psicológico en el trabajo. Santander: Sal Tarrea; 2001.

37. Vega V. Violencia, maltrato, acoso laboral: El mobbing como concepto. Buenos Aires; UBA, Lugar Editorial; 2008.

38. Universidad Nacional de Cuyo. Carreras de grado y pregrado [Internet]. Mendoza: UNCuyo [citado 10 dic 2015]. Disponible en: http://goo. $\mathrm{gl} / 3 \mathrm{wAJ} 7 \mathrm{~m}$.

39. Secretaría de Políticas Universitarias. Anuario de Estadísticas Universitarias: Argentina 2013 [Internet]. Buenos Aires: Ministerio de Educación de la Nación; 2013 [citado 10 ene 2016]. Disponible en: http://goo.gl/OKo3ep.
40. ADEMYS Asociación Docente, Taller de Estudios Laborales. Salud y Condiciones de trabajo en el sector docente: diagnóstico y respuestas posibles. Informe final provisorio marzo de 2011 [Internet]. Buenos Aires: ADEMYS, TEL; 2011 [citado $10 \mathrm{abr}$ 2015]. Disponible en: http://goo.gl/RBqH7w.

41. Sindicato Argentino de Docentes Privados. Las condiciones de trabajo y salud de los docentes privados [Internet]. Buenos Aires: SADOP; 2010 [citado 1 mar 2015]. Disponible en: http://goo.gl/ tUe2Cu.

42. Mendizábal N. Condiciones de trabajo y salud de los docentes primarios de la provincia de Buenos Aires. Buenos Aires: CEIL-PIETTE; 1995.

43. Dirección General de Estadística y Censos. Encuesta Anual de Hogares 2005: Uso del tiempo (Informe de Resultados No329) [Internet]. Buenos Aires: Gobierno de la Ciudad de Buenos Aires; 2007 [citado 20 mar 2015]. Disponible en: http:// goo.gl/bHpiVH.

44. Delfini M, Erbes A, Roitter S. Participación sindical de los trabajadores en Argentina: principales determinantes y perspectivas. Relations Industrielles. 2011;66(3):374-396.

45. Internacional de la Educación para América Latina. El desafío sindical: organización, estructura y fortalecimiento de sindicatos de la educación en América Latina. San José: IE; 2010.

46. Vidal S, Jodar P, Alós R. La relación entre afiliados y sindicatos, un análisis de la participación. Arxius de Ciencias Sociales. 2008;(18):39-54.

47. Secretaría de Gestión Administrativa, Económica y de Servicios, Coordinación de Gestión Administrativa, Dirección General de Medicina del Trabajo. Registro de Ausentismo de la UNCuyo (2012-2013). Mendoza: Universidad Nacional de Cuyo; 2013.

48. Neffa JC. Las condiciones y medio ambiente de trabajo: Presentación de la concepción dominante y de una visión alternativa. Buenos Aires: CEIL-PIETTE; 1995.

49. Abal Medina P. Ser sólo un número más: Trabajadores jóvenes, grandes empresas y activismos sindicales en la Argentina actual. Buenos Aires: Biblos; 2015.

50. Linhart D. Los asalariados y la mundialización: el caso francés. Sociología del Trabajo. 2002;(45):53-68.

51. López-Araújo B, Segovia AO, Peiró JM. El papel modulador de la implicación en el trabajo 
en la relación entre el estrés y la satisfacción laboral. Psicothema. 2007;19(1):81-87.

52. Lodahl TM, Kejner M. The definition and measurement of job involvement. Journal of Applied Psychology. 1965;(49):24-33.

53. Montes Cató JS, Pierbattisti D. Relaciones de poder y disciplinamiento en los espacios de trabajo: Un estudio sobre la dominación en empresas de telecomunicaciones. Estudios del Trabajo. 2007;(33):67-94.

54. Gómez M. Un modelo de análisis para entender las transformaciones del sindicalismo durante los 90 en la Argentina. Conflicto Social. 2009;2(2):98-135.

55. Loyo Brambila A. La investigación sobre sindicalismo docente: una reflexión sobre la diversidad de enfoques. El Cotidiano. 2011;(168):115-119.

56. Ministerio de Trabajo y Seguridad Social. La expansión de la afiliación sindical, análisis del módulo de relaciones laborales de la EIL [Internet]. Buenos Aires: Ministerio de Trabajo y Seguridad [citado 7 may 2015]. Disponible en http://goo.gl/ IhaVPs.

\section{NOTAS FINALES}

[a] La Universidad Nacional de Cuyo no cuenta, hasta el momento, con un Comité de Ética en Investigación (CEI), y las instituciones involucradas no plantearon como requisito que el proyecto de investigación fuera aprobado por un CEI.

[b] Se entiende por días caídos a aquellos en los cuales un trabajador/a no asistió a su lugar de trabajo y no pudo realizar sus tareas de manera habitual.

[c] La idea de "doble jornada" y de "doble presencia", mencionadas en el documento, corresponden al concepto "doppia presenza" introducido por Laura Balbo en 1978.

\section{FORMA DE CITAR}

Collado PA, Soria CB, Canafoglia E, Collado SA. Condiciones de trabajo y salud en docentes universitarios y de enseñanza media de Mendoza, Argentina: entre el compromiso y el desgaste emocional. Salud Colectiva. 2016;12(2):203220. doi: $10.18294 / \mathrm{sc} .2016 .710$.

Recibido: 19 de agosto de 2015 | Versión final: 9 de febrero de 2016 | Aprobado: 15 de marzo de 2016

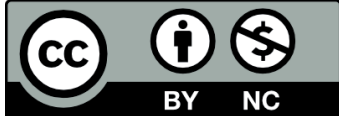

Este obra está bajo una licencia de Creative Commons Reconocimiento-NoComercial 4.0 Internacional. Reconocimiento - Permite copiar, distribuir y comunicar públicamente la obra. A cambio, se debe reconocer y citar al autor original. No Comercial - Esta obra no puede ser utilizada con finalidades comerciales, a menos que se obtenga el permiso. 